\title{
Retraction Note to: Inhibition of Lipopolysaccharide (LPS)-Induced Inflammatory Responses by Selenium in Bovine Mammary Epithelial Cells in Primary Culture
}

\author{
Zhengkai Wei, ${ }^{1}$ Minjun Yao, ${ }^{1}$ Yimeng Li, ${ }^{1}$ Zhengtao Yang, ${ }^{1}$ and Xiaosheng Feng ${ }^{2,3}$
}

Retraction to: Inflammation (2015) 38:152-158

DOI 10.1007/s10753-014-0017-9

The authors hereby retract the article entitled "Inhibition of Lipopolysaccharide (LPS)-Induced Inflammatory Responses by Selenium in Bovine Mammary Epithelial Cells in Primary Culture" (Wei et al. 2014) published in Inflammation, Volume 38 / Issue 1 (February 2015) because of inappropriate statistical analysis and inability to reproduce some of the results.

The online version of the original article can be found at http://dx.doi.org/ 10.1007/s10753-014-0017-9.

${ }^{1}$ College of Veterinary Medicine, Jilin University, Changchun, 130062, Jilin Province, China

${ }^{2}$ Guangdong Haid Institute of Animal Husbandry and Veterinary, Guangzhou, 510000, Guangdong, China

${ }^{3}$ To whom correspondence should be addressed at Guangdong Haid Institute of Animal Husbandry and Veterinary, Guangzhou, 510000, Guangdong, China. E-mail: fxs_2005@hotmail.com 